\title{
Bringing Reality into Calculus Classrooms: Mathematizing a Real-Life Problem Simulated in a Virtual Environment
}

\author{
http://dx.doi.org/10.3991/ijep.v3i1.2337 \\ O.V. Shipulina ${ }^{1}$, D. H. Smith ${ }^{2}$ and P. Liljedahl ${ }^{1}$ \\ ${ }^{1}$ Simon Fraser University, Burnaby, Canada \\ ${ }^{2}$ McMaster University, Hamilton, Canada
}

\begin{abstract}
The study explores how students, who had completed the AP calculus course, mathematized the optimal navigation real-life problem simulated in the Second Life Virtual Environment. The particular research interest was to investigate whether/how students' empirical activity in VE influences the way of their mathematizing.
\end{abstract}

Index Terms-Calculus, Realistic Mathematics Education, Virtual Environment.

\section{INTRODUCTION}

A troubling problem with current education is the practical application of knowledge. Graduates do not know how to apply knowledge to many problems that arise outside the walls of school [1]. A serious mismatch exists and is growing between the skills obtained at schools and the kind of understanding and abilities that are needed for success beyond school [2]. The problem of 'the practical application of knowledge' is especially significant for calculus, which has numerous applications, including a wide variety of engineering tasks.

The idea of including the out-of-school world in mathematics education, implying that focus be put on reallife applications, is not new and was emphasized in education policy in many countries [3-4]. Regarding teaching and learning calculus, in the late 1980s the Calculus Reform movement began in the USA. The Calculus Consortium at Harvard (CCH) was funded by the National Science Foundation to redesign the curriculum with a view of making calculus more understandable, more applied, and more relevant for a wider range of students. One of the desired characteristics of calculus course was that students and instructors would find the applications real and compelling [5]. Consequently, many teachers and textbook writers have been working on the development of mathematical school tasks that resemble out-of-school situations. Palm and Burman [6] reported that, in Finland and Sweden, in many of the tasks encountered by students in school mathematics the situation described in the task, is a situation from real life.

A traditional way of description of the contextualized tasks containing out-of-school real life situations is a so called 'word problems'. Word problems are firmly entrenched as a classroom tradition, particularly in North American schools [7]. And yet, there has been long lasting debates about the reasons for the lack of word problems' effectiveness as a link between abstract mathematics and real-life phenomena. Gravemeijer [8] notes that research on word problems has revealed the complex nature of the processes that lead to the lack of students' activation of their real-world knowledge. Palm [9] stresses that in a large number of studies students do not pay much attention to the realities of the situations described in the word problems. As such, students have a tendency not to make proper use of their real-world knowledge. Toom [10] believes that not all word problems are difficult in mathematical sense, but all of them need understanding of language and different modes of representation such as words, symbols, and images.

The contemporary computer technologies can provide much better simulations of real world situations in mathematical classrooms for connecting the mathematical abstract with out-of-school situations, which is a point of this research. It should be also mentioned that there exist the real-life situations which cannot be simulated on the computer screens and the word problem format may be the only way of their description. Our point is that balanced use of both, the word problem format and the computer simulations on the basis of an appropriate instructional design theory is a possible solution of the problem of practical application of knowledge.

The purpose of this study is to utilize Virtual Environment (VE) as a method of simulating real-life situations so that to bring the reality to the calculus classrooms. The task for the students was to find the optimal path in VE empirically, and then to transfer the simulated in VE reallife situation into a mathematical formal task. The particular research interest was to investigate whether/how students' empirical activity in VE influences their mathematical activity.

\section{THEORETICAL BACKGROUND}

More than forty years ago Freudenthal [11] posed the problem of lack of connection between mathematical knowledge and its real-life object. The Freudenthal Institute has developed a theoretical framework of Realistic Mathematics Education (RME) [11-13], which is based on Freudenthal's idea that mathematics must be connected to reality. The use of realistic contexts became one of the determining characteristics of RME approach to mathematics education. For Freudenthal mathematics was not the body of mathematical knowledge, but the activity of organizing matter from reality or mathematical matter, the activity called mathematization [14]. The most general characteristic of RME is mathematizing, wherein the 
realistic contexts must be used as a source for mathematizing.

According to Freudenthal [12], there is another important characteristic of RME closely related to mathematizing and which could be called the 'level principle'. The main idea of the principle is that students pass through different levels of understanding: from devising informal context-connected solutions to reaching some level of schematization. Finally they should have insight into the general principles behind a problem and should be able to see the overall picture. Essential for this 'level principle' is that the activity of mathematizing on a lower level can be the subject of inquiry on a higher level. One of the basic principles of RME is Freudenthal's view that mathematics is an activity, which is reflected in guided reinvention approach to teaching and learning.

Treffers [15] formulated the idea of 'progressive mathematizing' as a sequence of two types of mathematical activity - horizontal mathematizing and vertical mathematizing. He suggested that horizontal mathematizing consists of non-mathematical real world situations, transforming the situations into mathematical problems. Vertical mathematizing is grounded on horizontal mathematizing and includes reasoning about abstracts within the mathematical system itself. The process of extracting the appropriate concept from a concrete situation is stated by De Lange [16] as 'conceptual mathematization'. This process forces the students to explore the situation, find and identify the relevant mathematics, schematize, visualize, and develop a corresponding mathematical concept. By reflecting and generalizing the students will be able to apply the mathematical concept to new areas of the real world.

Another important aspect of RME is a special role of models. Particularly, models are seen as representation of problem situation and play the role of bridging the gap between the informal understanding (connected to the 'real world' and 'imagined reality') on the one side, and the understanding of formal systems on the other [17]. According to Streefland, cited in [14], models can fulfill the bridging function between the informal and the formal level: by shifting from a 'model-of' to a 'model-for'. At first, the model is a model-of a situation that is familiar to the students. By a process of generalizing and formalizing, the model eventually becomes an entity on its own. It becomes possible to use it as a model-for mathematical reasoning.

In the USA, RME was adopted in the 'Mathematics in Context' project whose aim was to develop a mathematics curriculum for the U.S. middle school. The project was funded by the National Science Foundation and executed by the Center for Research in Mathematical Sciences Education at the University of Wisconsin-Madison, and the Freudenthal Institute of Utrecht University. The philosophy of the curriculum and its development is based on the belief that mathematics, like any other body of knowledge, is the product of human inventiveness and social activities [14].

The RME theory has been accepted and adopted by some educational institutions of England, Germany, Denmark, Spain, Portugal, South Africa, Brazil, Japan, and Malaysia [16]. Regarding calculus, adaptations, development, and modification of RME instructional design theory for teaching and learning differential equa- tions are thoroughly performed in a number of publications, e.g.[18-22].

In spite wide acceptance and adaptation of RME, the recent research shows that there is still a wide gap between the world of knowledge obtained at school and the world of conceptions found in real-life experiences. The claim of this paper is that the reason of why students do not connect the mathematical world with reality is because they continue mathematizing only 'word problems' but not real-life situations which include the students' activities directed at the objects to be mathematized. Mathematizing a personal participation in a real-life situation may involve intuitive cognition, which in turn forms one of the basic components of mathematical activity along with formal aspects such as axioms, definitions, and algorithms [23].

\section{MATERIALS, Methods, AND PARTICIPANTS}

Second Life VE was used for programming an interactive setting for the empirical real-life optimal path finding task. The simulated setting includes a pond with shallow water and two platforms: one platform is located on land near the water's edge; another is located in the water (Fig.1).

The depth of the pond allows walking in water (instead of swimming) which in turn prevents from possible anxieties connected with absence of swimming skills. On the other hand, the depth of the pond allows feeling water resistance and therefore, speed difference in water and on land. The setting was programmed so that walking/running speed on land is twice as fast as walking/running speed in water. The Second Life VE allows utilizing both egocentric and allocentric view perspectives (Fig.1). Before completing each trip the participant has to reset the recorded from the previous trip data double clicking on the nearest pink flamingo. At the moments of leaving one green platform and stepping on another green platform there are the bell sounds informing about the beginning and finishing the trip.

The task for the student in this VE was to travel between the two green platforms trying to find the path which would give the shortest time of travel. The environment was programmed to record the time spent for each trip with a corresponding distance traveled by land and to display this information on the banners (Fig.1). After each trip the student had to transfer the data into a specially designed guiding-reflecting journal, which was an integral part of the instructional/experimental design (Fig. 2).
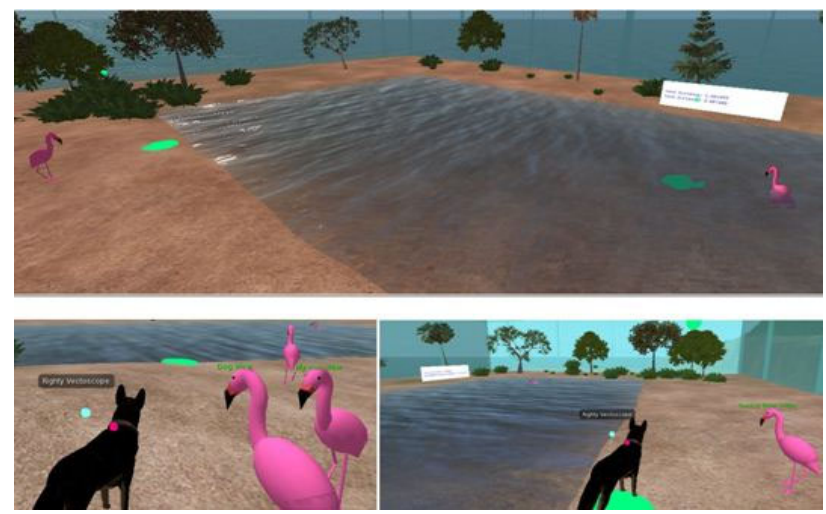

Figure 1. Simulated in the Second Life VE interactive setting. 

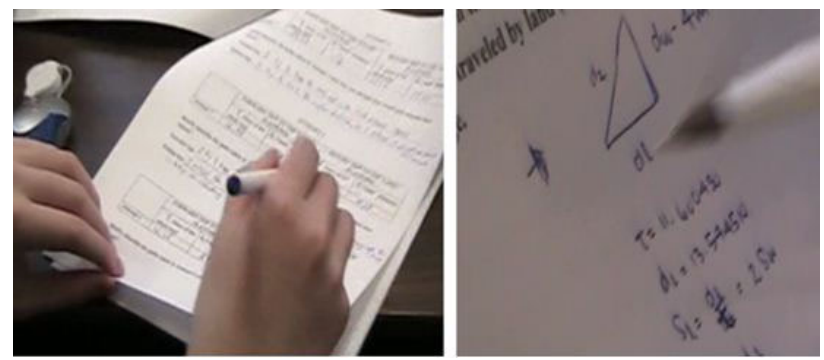

Figure 2. Working with guiding-reflecting journal.

The aim of the guiding-reflecting journal is to connect the student's optimal navigation practice in the VE with the calculus optimal path finding task, which in turn is available in almost every calculus textbook [24]. According to RME instructional design theory, the teacher provides guidance, playing a 'proactive role' within the classroom setting. In this study every student decides whether and to what extent he/she needs guidance. In other words, the students had a free choice: either to construct and develop their own models-of the situational problem or to accept and develop the journal's model. The journal contains blank space for independent reasoning and provides help/hints/directions for those students who need guidance and/or additional information.

The first part of the journal contains the instructions for VE activity followed by some information about water and land components of a path. After each trip the student had to describe briefly why he/she chose the particular trip. From the research aspect, these reflections could provide additional information for data analysis including the intuitive cognition impact. The journal provides tables for 10 attempts after which the student had to record the best (minimal) time out of these attempts with corresponding value of distance traveled by land. There is question in the journal about student's preferable path in case if the 'water' platform would be located closer to the beach. This question purposes to encourage student's reasoning about optimal path and platform location. The journal offers another 6 attempts of optimal navigation in the VE with new platform location. Then the journal suggests the student to try to solve the problem mathematically. At this point the student chooses whether to develop his/her own model-of the situational problem or to accept the model offered in the next page. The back page of the journal contains informational tips. From the research aspect, the back page location of the tips allowed to observe and videotape the particular moments when students used the provided information.

The model offered in the journal corresponds to the calculus optimal path finding task described in [24]. Namely, the task is to reach an object B, located in water, from the position A, located on land close to water edge, and to find such a path that would minimizes the time of travel from A to B (Fig. 3).

Path $\mathrm{AB}$ is the most direct and shortest, but also is of the longest water distance. Since the speed in water is slower than speed on land, the choice could be to use the shortest water distance which means sprinting down the beach to the point on shore closest to the 'water' platform, which is $\mathrm{C}$, and then turning a right angle and moving to B. Finally, there is the option of using a portion of the land path, up to $\mathrm{D}$, and then entering into the water at $\mathrm{D}$ and moving diagonally to the water platform.

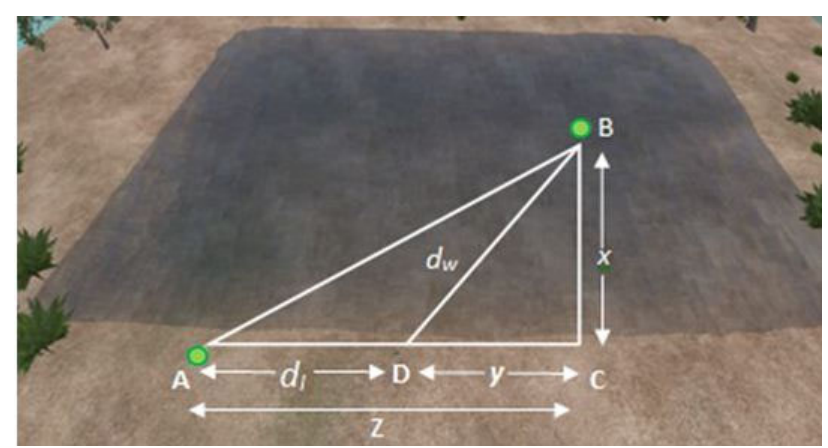

Figure 3. Possible paths: from location A on Land to location B in water.

Let $Z$ denote the distance between $\mathrm{A}$ and C. Let $y=z-$ $d_{l}$, and $X$ represents distance between B and C. Speed on land is $S_{l}$; speed in water is $S_{w}$. Then time spent for the trip is

$T=\frac{z-y}{s_{l}}+\frac{\sqrt{x^{2}+y^{2}}}{s_{w}}$

The condition of minimal time:

$T^{\prime}(y)=0$, or $\quad\left(\frac{z-y}{s_{l}}+\frac{\sqrt{x^{2}+y^{2}}}{s_{w}}\right)^{\prime}=0$

$T^{\prime}=\left(\frac{z-y}{s_{l}}+\frac{\sqrt{x^{2}+y^{2}}}{s_{w}}\right)^{\prime}=\left(\frac{z}{s_{l}}-\frac{y}{s_{l}}+\frac{1}{s_{w}}\left(x^{2}+y^{2}\right)^{\frac{1}{2}}\right)^{\prime}=-\frac{1}{s_{l}}+$

$+\frac{1}{s_{w}} \frac{1}{2}\left(x^{2}+y^{2}\right)^{-\frac{1}{2}} 2 y=0$

$\frac{y^{2}}{s_{w}^{2}\left(x^{2}+y^{2}\right)}=\frac{1}{s_{l}^{2}} ; y^{2}=\frac{s_{w}^{2}}{s_{l}^{2}} x^{2}+\frac{s_{w}^{2}}{s_{l}^{2}} y^{2}$

$y^{2}\left(1-\frac{s_{w}^{2}}{s_{l}^{2}}\right)=\frac{s_{w}^{2}}{s_{l}^{2}} x^{2}$

$y^{2}=\frac{s_{W}^{2}}{s_{l}^{2}} \frac{x^{2}}{\left(1-\frac{s_{W}^{2}}{s_{l}^{2}}\right)}=\frac{x^{2}}{\left(1-\frac{s_{W}^{2}}{s_{l}^{2}}\right) \frac{s_{l}^{2}}{s_{W}{ }^{2}}}=\frac{x^{2}}{\left(\frac{s_{l}^{2}}{s_{W}^{2}}-1\right)}=\frac{x^{2}}{\left(\frac{s_{l}}{s_{W}}-1\right)\left(\frac{s_{l}}{s_{W}}+1\right)} ;$

$y=\frac{x}{\sqrt{\frac{s_{l}}{s_{w}}+1} \sqrt{\frac{s_{l}}{s_{w}}-1}}$

The final solution (3) shows that the optimal path does not depend on $\mathrm{z}$, as long as $\mathrm{z}$ is larger than $\mathrm{y}$. There is no solution if $\mathrm{s}_{l}$ is smaller than $\mathrm{s}_{w}$.

The designed study contained three main stages. The exploration trial was the first stage of experimental design with unlimited duration until the student feels comfortable in the environment and announced that he/she was ready to start the next stage. The exploration trial allowed students to explore the pond with its shallow water and to feel the speed difference on land and in water. It also gave the students the opportunity to try egocentric and allocentric view perspectives and to choose the preferable one. Altogether, the goal of exploration trial was to let students get feeling of 'being' in the environment before starting the next, second, stage of the designed study which is an optimal navigation in the VE. The third stage of the designed study is mathematizing the VE activity which implies the journal work only. 
Students ranging in age from 17 to 18 years, who had almost completed the AP calculus course at a high school of Vancouver (Canada), participated in the research study. Each participant provided a signed Parent Consent Form. They also read and signed the Assent Form before participating. The experiments were conducted in the school's Teacher's room, outside of regular calculus class time. Each session lasted 60-90 minutes. The participants' exploration of the computer environment was screen recorded by SMR software. Their work with the journals was video-recorded.

\section{RESULTS}

The first participant, named Kenneth, performed fully independent, non-guided mathematizing. His VE activity was characterized by deliberate planning, realizing the trip strategies, and collecting empirical data for 'transforming a problem field into a mathematical problem' [15]. We call such activity 'empirical mathematizing'.

Kenneth spent 3.45 min exploring the environment moving in water and on land, therefore he had an opportunity to feel the speed difference in two different mediums. Moreover, he was informed by journal instructions and by the researcher that the speed on land is two times faster than the speed in water. Nevertheless Kenneth's first two trips were straight lines between the platforms which maximize lower speed water part of his path (Fig. 4).

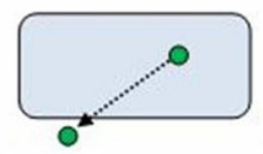

Figure 4. Kenneth’s first trip diagram.

For Kenneth it was self-evident, certain and intrinsic, that the shortest distance between two points should be a straight line. Obviously, Kenneth chose the straight line as a shortest distance having in mind an intuitive model that the shortest distance would give him the shortest time. Kenneth's tacit intuitive model prevailed over his knowledge about speed difference due to its robustness [25].

Remarkably, that already after two trips in VE Kenneth asked, “Actually, can I do math?” which means that his level of confidence in calculus application was very high and not typical for the school student [26]. At that moment Kenneth had in mind an implicit way of problem solving which can be referred to intuitive mathematizing from the viewpoint of its implicitness and globality, as described in [27]. Not relying on this intuitive approach, Kenneth decided to continue empirical mathematizing. Kenneth used the strategy of minimizing distance in water in third and fourth trips in VE. His best time strategy was the path between minimal and maximal water distances (Fig. 5).
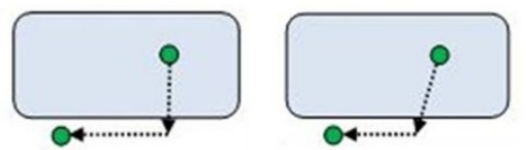

Figure 5. Kenneth's second and third strategies. The second is minimizing water distance (left diagram); the third strategy is Kenneth's best time trip (right diagram).

Altogether, Kenneth used three strategies and realized two trips (forward and return) using each of them. After testing all three strategies Kenneth decided that he had enough empirical knowledge and constructed his graphical model-of the situational problem which was fully grounded on his empirical activity (Fig.6).

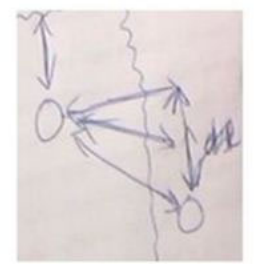

Figure 6. Kenneth's graphical model-of the problem.

Kenneth has a natural habit to think aloud which gave valuable additional information for data analysis. Particularly, while writing a relation in symbols between distances, speeds and time, Kenneth reasoned aloud and in 'real-life' terms. In other words, Kenneth was writing a formula containing abstract variables. According to Treffers's [15] classification, such activity relates to vertical mathematizing. On the other hand, while writing the formula, Kenneth articulated in terms of the variables' physical meanings. That means that the variables were attached to the physical reality in Kenneth's mind and as such, this activity can be also referred to horizontal mathematizing, or to border-line between them, or to vertical mathematizing intertwined with horizontal. Such interrelation agrees with Freudenthal's [12] viewpoint that distinction between horizontal and vertical activity is not rigid and structural. Then Kenneth transferred to vertical activity detached from horizontal manipulating with mathematical symbols. Interesting moment happened when Kenneth's vertical mathematizing resulted in "plus or minus" land distance, which obviously was a mathematical abstraction. This turned Kenneth back to situational horizontal layer for verification the plausibility of the obtained formal results.

Altogether, a few stages can be extracted from Kenneth's mental course: (a) from real-life situation via perception, intuitive cognition, and empirical mathematizing Kenneth shifts to the creation of a graphical model-of the situational problem in order to formalize it into the abstract mathematical idea. (b) He develops the graphical 'model-of' into a model-for mathematical reasoning. (c) Via progressive mathematization Kenneth goes to the formal mathematical world of vertical activity. (d) After utilization of some algorithms Kenneth turns back to situational physical world of horizontal layer to verify the plausibility of the obtained formal results.

In contrast to the first participant, the second student, named Jason, performed almost fully guided horizontal mathematizing. Starting from exploration trial, Jason spent less than 30 sec for it being in water during a very short time. He refused to explore the environment longer, so, obviously he didn't have an opportunity to feel the difference between speeds in water and on land. Nevertheless, he chose first trip strategy under the influence of information that speed on land was faster than speed in water (Fig.7).

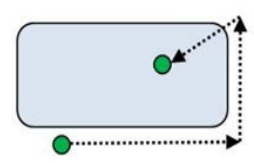

Figure 7. Jason's first trip diagram. 
Jason received this information before his first trip from the journal, and from the researcher. Jason commented this strategy as "Land is faster" and completed the next trip according to the same strategy of trying to minimize the distance in water (Fig.8).

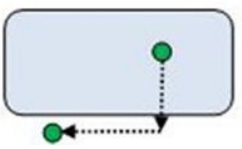

Figure 8. Jason’s second trip diagram.

Overall, Jason completed 10 trips the strategies of which, according to his comments, were either maximal or minimal water distances. The result of Jason's empirical mathematizing did not allow him to construct such modelof the situational problem, which would allow him to develop a model-for mathematical reasoning (Fig. 9).

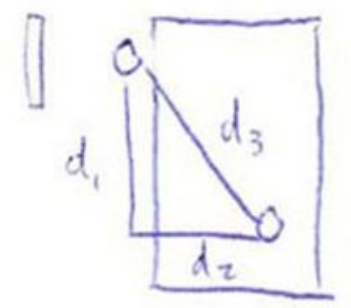

Figure 9. Jason's graphical model-of the problem.

Partly it happened because of the computer error, partly because of his limited choice of trip strategies. This right rectangle graphical model-of Jason's empirical activity was not a subject to further mathematical development because of absence of variables for mathematical exploration. As a result, Jason decided to develop the journal model. He demonstrated excellent independent vertical mathematizing which included application of the chain rule algorithm. Jason's mental course included the following stages: (a) From real-life situation via empirical mathematizing (which generated invalid empirical knowledge), to the creation of corresponding graphical model-of the situation. (b) Following the journal guidance of horizontal mathematizing. (c) Transferring to independent vertical activity.

The third student, named Nick, performed guided mathematizing which, from the viewpoint of the guidance, can be placed between the Kenneth's fully non-guided and Jason's guided activities. Nick spent 3.5 minutes for the exploration trial. He was the first participant who, during the exploration trial, tried to utilize both egocentric and allocentric view perspectives. Exploring the environment during 3.5 minutes Nick traveled in water and on land and had enough time to feel the speed difference in two mediums. Nevertheless, Nick's first trip was of the same strategy as the Kenneth's first trip-the strategy of a straight line between the platforms giving the shortest distance between the platforms but longest water distance (Fig.3). Both Kenneth's and Nick, prior to their first trips, knew from the journal instructions, from the researcher, and from their experience during the exploration trials, that the speed on land was faster than speed in water. But their tacit intuitive model that shortest distance should give the shortest time prevailed over knowing that the speeds were different in different mediums. The remark- able change in Nick's empirical mathematizing approach happened after 6 trips of different strategies, when he wrote in his comments, "I noticed the angle in which I enter the land from water is key in reducing the time". He planned all the other trips according to his new 'angle' approach.

Nick demonstrated how empirical mathematizing can result in the construction of his own original model-of the situational problem which he developed into model-for mathematical reasoning (Fig. 10).

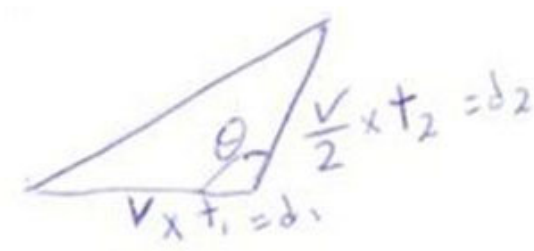

Figure 10. Nick's graphical model-of the problem.

Nick was persistent in mathematical verification of his empirical finding. He spent more than 10 minutes working on his original model independently, and another 6 minutes working on the journal model development. Notably, during this last 6 minute period Nick continued to write on the page containing his own model, which indicates that he tried to keep some independence from the journal guidance. He performed all stages of mathematizing: from empirical to horizontal, grounded on empirical; from horizontal to vertical grounded on horizontal.

All three students demonstrated the crucial role of empirical mathematizing in construction of the models-of the situational problem. During empirical activity all three participants demonstrated the interference of intuitive cognition on planning some trip strategies. Particularly, Kenneth's and Nick's first trip strategies were determined by tacit intuitive model which prevailed over newly received knowledge about speed differences in different mediums. This agrees with tacit intuitive models' fundamental characteristic of robustness. On the other hand, all three participants demonstrated that empirical knowledge obtained from their empirical mathematizing prevailed over intuitive cognition/intuitive models and fully determined the models-of the situational problem. This in turn may suggest that new empirical activity forms new empirical knowledge which in turn either agrees with the intuitive cognition formed on the basis of previous empirical knowledge or prevails over it and forms new intuitions. If students mathematize the real-life problem presented in the format of word problem, the intuitive cognition based on their previous empirical experience may play the prevailing role at the stage of horizontal mathematizing. If students are provided with opportunity for empirical mathematizing, their new empirical knowledge prevails over intuitions; their horizontal mathematizing is fully grounded on empirical mathematizing.

The students' ability to mathematize the situational problem with or without guidance depends on their stage of knowledge, according to the epistemological empowerment model, described by Ernest [26]. Particularly, Kenneth demonstrated the Constructed Knowledge Stage, characterized by confidence for integrating Connected Knowing (the intuitive knowing) and Separated Knowing (the impersonal rational reasoning). Being a constructing 
knower, Kenneth was confident in mathematical knowledge and in the ability to apply his knowledge. He also had a sense of mathematical self-efficacy. Unlike Kenneth, Jason's epistemological empowerment relates to the earlier, Separate Knowing stage. This earlier stage of knowing is a rational mode in which the subject realizes that there are objective logical rules, impersonal rational reasoning and uses them. Nick's confidence in developing his own model allows relating his empowerment to the Constructed Knowledge stage. On the other hand, at some point Nick lost his confidence and decided to develop the journal model. Therefore, his epistemological empowerment may correspond to the stage between the Connected Knowing and the Constructed Knowledge.

\section{CONCLUSIONS}

We demonstrated that instead of real-life situations described by 'word problems' with ready-made images to be mathematized, the real-life activity can be simulated in VE. A background assumption which was made at the beginning of the research was that the VE technology, and particularly the Second Life VE provides simulation on the computer screens close to the reality; and the real-life problems simulated in VE can be considered as the problems of the real physical world. The fact that all three students developed their models-of the situational problem on the basis of their empirical activity in VE suggests that VE indeed provides simulation close to reality; close enough to meet the purpose of this study, to bring the reallife problem from outside the school into the classroom. This, in turn, contributes to fundamental principle of RME by making formal mathematics as a natural extension of students' experiential reality. Another contribution to $\mathrm{RME}$ is connected with identifying the role of empirical mathematizing and empirical knowledge in construction of the models-of the situational problems and as such, in horizontal and vertical mathematizing.

Interesting finding concerns the role the intuitive cognition plays on different stages of mathematizing. Particularly, on the basis of the research results it was suggested that new empirical knowledge obtained from empirical mathematizing prevails over intuitions formed from a previous experience.

We also showed that the way of mathematizing depends on the stage of epistemological empowerment which is especially important for the ability to apply calculus for the beyond the school tasks, including a variety of engineering problems. Therefore, the instructional design based on utilization of VE simulations should develop students' epistemological empowerment through the development of their applicable skills.

VE environment simulations of real-life problems can be used for a variety of mathematics and science problems, preparing students for their future engineering careers, implying application of theoretical knowledge for practical tasks.

\section{REFERENCES}

[1] E.V. Ilyenkov, The Ideal in Human Activity. New York: Lawrence \& Wishart, 2009.

[2] R. Lesh and J. S. Zawojewski, "Problem solving and modeling," in Second Handbook of Research on Mathematics Teaching and Learning, F. K. Lester, Ed. Greenwich, CT: Information Age Publishing, 2007, pp. 763-805.
[3] T. Palm, " Word problems as simulations of real-world situations: A proposed framework," For the Learning of Mathematics, vol. 26, pp. 42-47, 2006.

[4] T. Palm, "Theory of authentic task situations," in Modelling Verbal Descriptions of Situations, L. Verschaffel, B. Greer, W. Van Dooren and S. Mukhopadhyay, Eds. Rotterdam: Sense Publishers, 2009, pp. 3-17.

[5] D. O. Tall, D. Smith and C. Piez, "Technology and calculus," in Research on Technology and the Teaching and Learning of Mathematics: Research Syntheses, M. K. Heid and G. W. Blume, Eds. Charlotte: Information Age Publishing, 2008, pp. 207-258.

[6] T. Palm and L. Burman, "Reality in mathematics assessment: an analysis of task-reality concordance in Finnish and Swedish national assessments " Nordic Studies in Mathematics Education, vol. 9, pp. 1-38, 2004.

[7] S. Gerofsky, "A linguistic and narrative view of word problems in mathematics education " For the Learning of Mathematics, vol. 16, pp. 36-45, 1996.

[8] K. Gravemeijer, "Commentary solving word problems: a case of modelling? " Learning and Instruction, vol. 1, pp. 389-397, 1997. http://dx.doi.org/10.1016/S0959-4752(97)00011-X

[9] T. Palm, "Impact of authenticity on sense making in word problem solving " Educational Studies in Mathematics, vol. 67, pp. 37-58, 2008. http://dx.doi.org/10.1007/s10649-007-9083-3

[10] A. Toom, "Word problems: Applications or mental manipulatives " For the Learning of Mathematics, vol. 19, pp. 36-38, 1999.

[11] H. Freudenthal, "Why to teach mathematics so as to be useful?" Educational Studies in Mathematics, vol. 1, pp. 3-8, 1968. http://dx.doi.org/10.1007/BF00426224

[12] H. Freudenthal, Revising Mathematics Education. Dordrecht, The Netherlands: Kluwer Academic, 1991.

[13] K. P. E. Gravemeijer, Developing Realistic Mathematics Education. Utrecht: CD-b Press / FreudenthalInstitute, Utrecht University, 1994.

[14] M. Van den Heuvel-Panhuizen, "The didactical use of models in realistic mathematics education: An example from a longitudinal trajectory on percentage " Educational Studies in Mathematics, vol. 54, pp. 9-35, 2003. http://dx.doi.org/10.1023/B:EDUC.00 00005212.03219.dc

[15] A. Treffers, Three Dimensions: A Model of Goal and Theory Description in Mathematics Instruction. The Wiskobas Project. Dordrecht: Kluwer Academic Publishers, 1987.

[16] J. De Lange, "Using and applying mathematics in education," in International Handbook of Mathematics Education, A. J. Bishop, K. Clements, C. Keitel, J. Kilpatrick and C. Laborde, Eds. Dordrecht, The Netherland: Kluwer Academic Publisher, 1996, pp. 49-97.

[17] K. Gravemeijer, "How emergent models may foster the constitution of formal mathematics " Mathematical Thinking and Learning, vol. 1, pp. 155-177, 1999. http://dx.doi.org/10.1207/ s15327833mtl0102_4

[18] O. N. Kwon, "Conceptualizing the realistic mathematics education approach in the teaching and learning of ordinary differential equations " in Proceeding of the Second International Conference on the Teaching of Mathematics (ICTM2), Greece, 2002.

[19] C. Rasmussen and K. King, "Locating starting points in differential equations: A realistic mathematics approach " International Journal of Mathematical Education in Science and Technology, vol. 31, pp. 161-172, 2000. http://dx.doi.org/10.1080/0020 73900287219

[20] C. Rasmussen and K. Marrongelle, "Pedagogical content tools: Integrating student reasoning and mathematics in instruction " Journal for Research in Mathematics Education, vol. 37, pp. 389420, 2006.

[21] C. Rasmussen, M. Zandieh, K. King and A. Terro, "Advancing mathematical activity: A practice - oriented view of advanced mathematical thinking " Mathematical Thinking and Learning, vol. 7, pp. 51-73, 2005. http://dx.doi.org/10.1207/s153 27833mt10701 4

[22] M. Stephan and C. Rasmussen, "Classroom mathematical practices in differential equations " The Journal of Mathematical 
PAPER

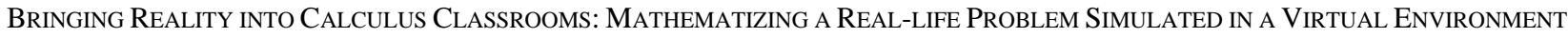

Behavior, vol. 21, pp. 459-490, 2002. http://dx.doi.org/ 10.1016/S0732-3123(02)00145-1

[23] E. Fischbein, "The interaction between the formal, the algorithmic, and the intuitive components in a mathematical activity," in Didactics of Mathematics as a Scientific Discipline, R. Biehler, R. W. Scholz, R. Strässer and B. Winkelmann, Eds. Dordrecht: Kluwer Academic Publishers, 1994, pp. 231-245.

[24] T. J. Pennings, "Do dogs know calculus?" The College Mathematics Journal, vol. 34, pp. 178-182, 2003. http://dx.doi.org/10.2307/ 3595798

[25] E. Fischbein, "Tacit models and mathematical reasoning " For the Learning of Mathematics, vol. 9, pp. 9-14, 1989.

[26] P. Ernest, "Empowerment in mathematics education," Philosophy of Mathematics Education Journal, pp. 1-16, 2002.

[27] E. Fischbein, Intuition in Science and Mathematics. Dordecht, The Netherlands: Reidel, 1987.

\section{AUTHORS}

O. V. Shipulina is with Faculty of Education, Simon Fraser University, Burnaby, BC V5A 1S6, Canada (email: oshipuli@sfu.ca).

D. H. Smith is with the Department of Communication and Multimedia, McMaster University, Hamilton, ON L8S 4L8, Canada (e-mail: dhsmith@mcmaster.ca).

P. Liljedahl is with Faculty of Education, Simon Fraser University, Burnaby, BC V5A 1S6, Canada (e-mail: liljedahl@sfu.ca ).

Received 1 November 2012. Published as resubmitted by the authors 18 December 2012. 